\title{
CONCEPTUALIZACIÓN DE OCUPACIÓN EN LOS CADERNOS BRASILEIROS DE TERAPIA OCUPACIONAL
}

\author{
CONCEPTUALIZATION OF OCCUPATION IN BRAZILIAN JOURNAL OF \\ OCCUPATIONAL THERAPY
}

\section{Rodolfo Morrison Jara', Enrique Henny Koller², Silvia Gómez Lilo 3}

\begin{abstract}
RESUMEN
Contexto: La conceptualización del constructo ocupación en la Terapia Ocupacional Latinoamericana es muy diversa yvaría dependiendo de la región y el idioma. Objetivo: la presente revisión bibliográfica narrativa pretende dar cuenta del uso y la conceptualización del constructo ocupación en la revista científica de Brasil Cadernos Brasileiros de Terapia Ocupacional (antiguamente nombrada como Cadernos de Terapia Ocupacional de la Universidad Federal de San Carlos). Método: se emplea una revisión bibliográfica narrativa, seleccionando artículos que emplearon el término ocupación en el título, el resumen o dentro de las palabras clave desde los primeros artículos disponibles en formato on line hasta el año 2014. Resultados: siete artículos discuten el concepto de ocupación de forma teórica o práctica. Conclusiones: Se observa que existe una gran diversidad en el uso del constructo y que, por lo general, se cuestiona su pertinencia cultural en el contexto brasileño.
\end{abstract}

PALABRAS CLAVE

Actividad, Brasil, Ciencia de la Ocupación, Ocupación, Terapia Ocupacional

\begin{abstract}
Context: The conceptualization of construct of occupation in Occupational Therapy in Latin America is diverse and varies depending on the region and language. Objective: This narrative review aims to give an account of use and conceptualization of occupational construct in scientific journal Brazilian Journal of Occupational Therapy. Methodology: a narrative review is used, selecting articles that used term occupation in title, abstract or within keywords until the year 2014. Results: seven articles discuss the concept of occupation in a theoretical or practical way: It is observed there is a great diversity in use of this construct, and generally its cultural relevance in the Brazilian context is criticized.
\end{abstract}

\section{KEYWORDS}

Activity, Brazil, Occupational Science, Occupation, Occupational Therapy

Recibido: $13 / 06 / 2017$

Aceptado: 05/06/2018

1 Dr. en Lógica y Filosofía de la Ciencia (Univ. de Salamanca); Terapeuta Ocupacional y Lic. en Ciencias de la Ocupación (Univ. Austral de Chile). Académico de la Universidad de Chile, integrante de la Sociedad Chilena de Ciencia de la Ocupación, del Comité de Estudios en Ciencia de la Ocupación (COECo) de la Universidad de Chile y del Núcleo Interuniversitario de Investigación Estudios Críticos en Diversidad. Avda. Independencia 1027, Santiago de Chile. Tel: +5629786545. Google Scholar: https://goo.gl/c72gPy; ResearchID: I-7124-2016; orcid.org/0000-0002-2834-1646. Contacto: rodolfomorrison@med.uchile.cl

2 Terapeuta Ocupacional, Licenciado en Ciencia de la Ocupación de la Universidad de Chile. Investigador invitado Comité de Estudios de Ciencia de la Ocupación,. Miembro del Colegio de Terapeutas Ocupacionales de Chile y de la Corporación Chilena de Integración Sensorial. Ex Vicepresidente de la Federación Mundial de Terapeutas Ocupacionales. Centro de Terapias para el Desarrollo Ltda. Correspondencia a: Avda. Independencia 1027, Santiago de Chile. Tel: +5629786545. Santiago de Chile. ResearchID: E-4987-2017; orcid. org/oooo-ooo3-4345-9658; Google Scholar https://goo.gl/P93oMc. Contacto: enrique.henny@gmail.com

3 Terapeuta Ocupacional, Licenciada en Ciencia de la Ocupación; Académica de la Universidad de Chile; integrante de la Sociedad Chilena de Ciencia de la Ocupación y del Comité de Estudios en Ciencia de la Ocupación (coeco). Avda. Independencia 1027, Santiago de Chile. Tel: +5629786545. Contacto:sgomez@med.uchile.cl 


\section{INTRODUCCIÓN}

La conceptualización del constructo ocupación en la Terapia Ocupacional Latinoamericana es muy diversa y varía dependiendo de la región y el idioma. Por ello, esta revisión da cuenta del uso y la conceptualización del constructo ocupación en la revista científica $\mathrm{Ca}$ dernos Brasileiros de Terapia Ocupacional (antiguamente nombrada como Cadernos de Terapia Ocupacional de la Universidad Federal de San Carlos), una de las revistas con mayor número de publicaciones de Brasil y Latinoamérica. Si bien la cantidad de artículos encontrados es escasa, la profundidad del análisis y resultados de cada uno es muy interesante para el desarrollo latinoamericano de la profesión.

La motivación por realizar esta revisión surge como parte de una investigación mayor que buscó identificar los usos del constructo ocupación en Latinoamérica. En esa línea otro artículo ha sido publicado respecto a los usos del constructo en la Revista Chilena de Terapia Ocupacional (Morrison, Gómez, Henny, Tapia \& Rueda, 2017).

\section{MÉTODO}

El método empleado corresponde una revisión narrativa (Aguilera, 2014). La que consiste en la selección de escritos según la experiencia de los autores o autoras empleando unos términos de búsqueda determinados, pero sin la exhaustividad que caracteriza a una revisión sistemática, por ejemplo. Así, se emplea la descripción de las ideas centrales en los hallazgos que a criterios de los/as autores/as resultan relevantes para el tema que se requiera. De esta manera, se seleccionaron artículos desde las primeras publicaciones disponibles en línea por la revista hasta el año 2014, empleando el término ocupación en el título, el resumen o en las palabras clave. Del universo de artículos a la fecha, se seleccionaron 7 , que discuten el concepto de ocupación de forma teórica o práctica.

\section{Resultados}

En su artículo acerca del uso de actividades de tiempo libre en terapia ocupacional (TO) Martinelli (2011) em- plea el concepto de ocupación como sinónimo de actividad y que ésta se encuentra presente en la vida de cualquier persona; y que es imposible pensar el ser humano sin las actividades. Emplea el concepto de ocupación como sinónimo de actividad la cual está presente en la vida de cualquier persona, vinculada a diversas funciones, como trabajo u ocio. Señala que es imposible pensar en el ser humano sin el uso de actividades.

Estima que en la TO todas las actividades humanas surgen por una necesidad individual para producir y llevar a cabo el hacer. Todas las actividades ocurren por una razón y tienen una explicación. Considera que las concepciones de las y los terapeutas ocupacionales están relacionadas con sus modelos teórico-prácticos.

Aunque cada profesional tiene una forma de actuary una comprensión de la ocupación humana, MartineIli hace hincapié en la necesidad de entender la actividad de manera más amplia, que permite identificar en las personas, sus valores, en tanto dueñas de sus acciones y representaciones en sus vidas. En su opinión, esta reflexión en la búsqueda del conocimiento de sí mismo lleva a la realización personal y a la participación activa de las personas, siendo una estrategia utilizadas por la terapeuta ocupacional al ver la actividad de manera amplia.

Para Martinelli, la profesión de TO, debido al nombre y su historia, lleva una marca asociada que consideraría la ocupación sólo como ocupación de un tiempo. Sin embargo, con el desarrollo e investigaciones en la profesión, se comprende que esta ocupación del tiempo es una elección personal, y el gran mérito de la TO es favorecer una reflexión, de manera que la persona pueda encontrar un sentido a este hacer u ocupación.

De esta manera, la autora propone que la TO no debe aferrarse al pasado y considerar al ocio desde prejuicios. El o la terapeuta ocupacional debe entender que se puede usar cualquier actividad, en tanto es un instrumento de su acción profesional.

Así, el ocio puede constituirse como un instrumento o medio de ocupación, pero pocos reconocen su valor como un estímulo para el desarrollo personal y un facilitador para la inclusión social de personas con discapacidad. 
Finalmente, Martinelli llama a romper etiquetas asociadas a las actividades como poco importantes. La TO hace una lectura del ocio, que contribuye al autoconocimiento de las personas con discapacidad y la población general. Más allá del autoconocimiento, observar estas actividades ofrece pistas de dificultades o habilidades en diferentes funciones que conforman el desarrollo humano.

Por otro lado, las autoras Stoffel y Nickel (2013), en su artículo acerca del uso de actividades en TO, en rehabilitación neurológica, refieren al Modelo de Ocupación Humana, desarrollado por Kielhofner, Burke y Heard desde el año 1980, con el fin de articular los conceptos implicados en su práctica. En este modelo, la ocupación humana se caracteriza por la relación dinámica entre los subsistemas de la volición, habituación y capacidades de desempeño de las personas y que está influenciada por el entorno del sujeto.

En otro línea, Araújo, Guimarães y Di Napoli (2013) discuten los conceptos de ocupación acción, hacer y actividad en la literatura brasileña. Refieren que les motivó la necesidad de profundizar la comprensión de estos términos relacionados con la TO. Señalan que Joe Benetton en el año 2008 indicó diferencias entre los conceptos de actividad humana, acción, ocupación, actividad ocupacional e incluso ejercicios; señalando que las terapeutas ocupacionales han transitado en la construcción colectiva de estas concepciones.

Las autoras revisan y destacan discusiones que buscan distinguir los términos actividad y ocupación, una disputa que tiene resonancias con la discusión presentada anteriormente y que ha sido formulada por terapeutas ocupacionales angloparlantes.

En la misma línea, Lima, Loureiro, Bentes, de Lima y Costa (2013) discuten acerca del valor terapéutico de la acción del hombre revisando la literatura. Mencionan a Kielhofner (2002), quien señala que el cuerpo humano tiene un principio ocupacional inherente. Este principio se manifiesta en las diferentes formas de hacer, ya sea en el trabajo, juego o actividades de la vida diaria, con un contexto temporal, físico y social que caracteriza en gran medida la vida humana.

Estas autoras proporcionan uno de los artículos más interesantes en cuanto a la diversidad de conceptos de ocupación. Indican que buscaron la comprensión de las diversas formas en que se ha abordado en TO la búsqueda de soluciones al problema del valor terapéutico de la acción humana, en América del Sur y del Norte, en particular Brasil, Canadá y Chile. Proponen así, entender las diferencias entre actividad y ocupación, desde diferentes aproximaciones teóricas.

Pero aclaran que, incluso entre los terapeutas ocupacionales, todavía no hay acuerdo sobre el significado de los términos centrales de la profesión. En cuanto a algunas teóricas de la Ciencia de Ocupación chilena, actividad significa un término genérico que se refiere a la característica humana de estar siempre activo. Esta es una idea arraigada en las mentes de las personas y la cultura compartida. El resultado de la interacción entre la actividad, la persona y el medio ambiente, de la que surgen forma, función y significado, es la ocupación, una actividad con significados subjetivos atribuidos por alguien como resultado de su experiencia única en el mundo (Carrasco y Olivares, 2008; Gómez, 2003). Para ellas, la terapeuta ocupacional trabaja en la forma, propósito y significado ocupación inherente. La forma se refiere a la actividad de la interacción y el medio ambiente, lo que resulta en el espacio físico real en el que se dio cuenta de la idea. El objetivo tiene que ver con la interacción entre la persona y la actividad, que es por qué se hace algo. Los resultados y significados de la interacción entre la persona y el medio ambiente (Carrasco y Olivares, 2008; Imperatore y Henny, 2002; Gómez, 2003; Rueda, 2003).

También está el uso de término praxis, tanto para las actividades que tienen lugar durante el proceso y el objetivo final de la intervención. Francisco (2004) define la práctica como una actividad, como un producto de la conciencia, que cambia la realidad. Para ella, es la actividad desarrollada en el lugar de la TO (en cuanto a lo que el terapeuta quiere que suceda al final del proceso), la vida del sujeto. Caniglia (2005) considera que el término se refiere a la propia actividad humana más que ocupación o trabajo, prefiriendo hablar de la práctica en la profesión.

Las autoras Lima, Loureiro, Bentes, de Lima y Costa (2013) señalan que se han percatado de las dificultades para llegar a un consenso sobre el uso del término "ocupación" y que la TO tiene que esforzarse para superarlo. Sin embargo, a su entender, hablar de la actividad u ocupación no es un tema que se resolverá en la Ciencia de la Ocupación (CO). 
Finalmente, señalan que han mantenido prejuicios en la manera de hablar de ocupación, de manera similar a Araújo, Guimarães y Di Napoli (2013), vinculando ocupación a ocio, asociándola a un ámbito de poca importancia y limitada al hacer por el hacer, o para pasar el tiempo.

Las autoras concuerdan con las teóricas de la CO, señalando la importancia de buscar fundamentos científicos sobre este concepto central en la disciplina. También, señalan que ha existido miedo en Brasil para hablar de la ocupación.

Reconocen que han existido cambios en la manera de entender ocupación, desde el sentido reduccionista hacia la deconstrucción del concepto; hasta basarse en la fenomenología y en una visión integral del ser humano y del mundo, donde las contribuciones son tan importantes como necesarias para la profesión.

De esta manera, hablar de forma, función y significado en las ocupaciones es un aspecto que opera en el cambio de la realidad del sujeto. Están de acuerdo con las autoras chilenas al diferenciar dos dimensiones de la acción humana. En una de ellas, de manera más general, no se puede observar lo que se refiere a una característica humana de estar siempre en el camino, una idea culturalmente arraigada. La otra resulta de la interacción del individuo con el mundo que le rodea, estableciendo un significado, lo que sucede durante el proceso de la TO y que es, también, el foco de la profesión.

Como resultado, las autoras añaden los significados atribuidos a la ocupación, la comprensión de lo más cerca a lo desarrollado para la actividad. No se encontraron conflictos en el uso de ambos conceptos. Por ello, concluyen que la TO no debe ser una mera defensa de un modelo, ya que la materia teórica de la profesión es compleja y, por lo tanto, se requieren diferentes entendimientos. La/el terapeuta ocupacional debe aprender a vivir con la diversidad de conceptos, tratando de integrarlos. Por lo tanto, conocerlos, formar su identidad y aprender a andar su propio camino, creer en lo que hace.

En otro artículo, Magalhães (2013) propone contextualizar los conceptos de ocupación y la actividad desde la perspectiva de la literatura contemporánea de la TO y la CO. Para esto, recurre al material publicado en inglés en las últimas décadas, pero asumiendo su posición al respecto. Reconoce en su experiencia, como profesora e investigadora brasileña que trabaja desde hace una década en Canadá, como la influencia en su percepción, pero señala que su intención es proporcionar una visión general de las principales tendencias y tensiones en estos conceptos claves de las dos áreas.

De esta manera, comienza señalando que en el centro de este debate se encuentra el nombre mismo de la profesión: TO. Como muchos han señalado, la ocupación lleva significados dudosos en inglés y no siempre se traduce adecuadamente a otros contextos lingüísticos (Magalhães \& Galheigo, 2010; Nelson y Jonsson, 1999).

Este ajuste muestra un valor característico: la adopción del concepto de significado, que es muy relevante para la mayoría de las publicaciones relacionadas con el área. De hecho, la preponderancia de lo que significa en la calidad de la ocupación humana que se genera la expresión, haciendo.

El tema de la ocupación como un factor determinante de la salud se refiere en varias obras, tanto en el área de la ciencia, como en la práctica de la TO. Por lo tanto, el establecimiento de una estrecha relación entre el significado, el hacer y el ser, junto con el procesamiento y la salud, justifica la aparición de modelos de análisis de intervención de una ocupación que ahora se califica como una condición sine qua non de la inclusión social, de la salud y el bienestar (Wilcock, 2007).

En esta perspectiva, no todas las ocupaciones serían beneficiosos para la salud, sólo aquellas que tienen una importancia innegable para un individuo o una comunidad. Vale la pena señalar, sin embargo, que se ha criticado al predominio de una visión optimista de la ocupación humana, es decir, aquellos que argumentan que las ocupaciones son siempre beneficiosas para la salud, que es bastante común en la literatura profesional (Kiepek \& Magalhaes, 2011 ).

La autora continúa con este análisis indicando que las barreras del idioma en la estandarización de terminologías profesionales son ampliamente conocidos y no serían diferentes en el trabajo de la TO (Magalhães \& Galheigo, 2010). El concepto de ocupación se muestra numerosas veces de forma contradictoria (Pierce, 2001; Royeen, 2002). Además, refiere a Kantartzis y Molineux (2012) para señalar que el término se ha basado principalmente en supuestos que se articulan a partir de 
un estilo de vida asociado con ideologías de varones blancos de clase media y marcada por los valores hegemónicos en los países occidentales. A su vez, cita a Ramugondo y Kronenberg (2015), quienes admiten que una definición universal de ocupación es poco probable, pero sostienen que hay que buscar la pluralidad de perspectivas culturales, incluyendo ontologías y epistemologías del Sur. No obstante, la Sociedad Internacional de la Ciencia Ocupacional utiliza términos muy generales para definir la ocupación.

Por lo tanto, la distinción entre la ocupación como un fin o meta final de la intervención terapéutica y la ocupación como una forma o medios de intervención, está en el centro del debate $y$, en cierto sentido, en el corazón de los cambios en los modelos propios acordados.

Magalhães (2013) señala que es razonable suponer que varias condiciones pueden haberse para la vulnerabilidad conceptual señalada por muchos en la TO y la CO. Estudiosos sostienen, por ejemplo, que usar la palabra como sinónimo de actividad, ocupación trae numerosos problemas y necesita ser eliminado (Nelson, 1997) o con diligencia restablecerlo (Pierce, 2001). De hecho, la expresión de la actividad ha sido, si no se evita, al menos marginado en los documentos oficiales, como se muestra en este extracto de la última definición oficial de la Federación Mundial de Terapeutas Ocupacionales.

De esta forma, la autora señala que, lejos de ser espontánea, la sustitución progresiva de la palabra actividad por ocupación, término en los documentos internacionales de orientación tanto a la enseñanza y la práctica de los terapeutas ocupacionales, al menos de habla inglesa, no sólo se ha planeado, sino también, acompañado de una intensa actividad la investigación y el debate.

A pesar de cierta ambigüedad, la expresión ocupación se convierte en un punto de partida para el desarrollo posterior de la base científica de la profesión, consolidada a partir de dos estrategias principales: el modelo de intervención basado en la evidencia (Law y Baum, 1998) y el estudio sistemático de la complejidad de la ocupación humana y su uso en los procesos terapéuticos y el cambio social (Prodinger y Stamm, 2012).

Finalmente Magalhães (2013) concluye señalando que los terapeutas ocupacionales se enfrentan a la extraordinaria tarea de maximizar las oportunidades laborales de las personas y grupos con los que trabajan, a partir del reconocimiento del enorme potencial de la ocupación creativa, significativa y transformadora. Y espera que su trabajo pueda componer un diálogo abierto, no sólo en la trayectoria conceptual de ocupación y actividad, sino también en diversos contextos nacionales e internacionales, y abrir posibilidades prácticas de las asociaciones intelectuales y técnicas que conducen a la transformación de las bases de nuestras prácticas a través de la comprensión de su complejidad.

Una mirada diferente es la que refieren Da Silva y Dornelas (2014), posicionándose desde la literatura brasileña. Indican que para la TO el término ocupación se utiliza "para capturar el tamaño y la importancia de la actividad de todos los días" (Carleto et al., 2010, p. 61).

Y por último, para finalizar esta revisión, Monteiro, Ferreira, Cavaleiro y Costa (2014) realizan una aplicación clínica a la ocupación. Las autoras señalan que para algunos investigadores en el campo de la CO, la ocupación se define como unidades de acción continua, deliberada y conscientemente ejecutadas. Además, las ocupaciones tienden a ser significativas en el contexto de vida de las personas. En este campo de trabajo, la ocupación es considerada el foco central del ejercicio de la práctica de la TO, así como la unidad de análisis de esta ciencia (Larson, Wood\& Clark, 2005). Refieren a: Gómez (2006), señalando que la ocupación implica un hacer diario en el que las personas están involucradas y que la ocupación tiene formas, funciones y significados definidos y observados en un contexto cultural; a Torrico, Urrutia y Schliebener (2008) para indicar que el significado es el término utilizado para etiquetar la interpretación individual de la ocupación, se refiere al sentido de la percepción y las asociaciones cognitivas de cada individuo y que ése es uno de los conceptos que más caracteriza a la terminología de la ocupación. En este sentido, para la $\mathrm{CO}$ el significado se define como la experiencia subjetiva de la participación en ocupaciones y la participación de varios factores.

Por lo tanto, las ocupaciones en las que las personas se involucran en la actualidad pueden considerarse como parte de un proceso vinculado a la necesidad por ocuparse por mediante la modificación del significado de la situación en la que se encuentran (Echeverría, Ortega y Riquelme, 2009). Su investigación es pionera en la comprensión de la ocupación humana con personas que han experimentado quemaduras. Desde esta perspectiva, se entiende que la $\mathrm{TO}$ anclada en la $\mathrm{CO}$ 
puede analizar cómo los humanos entienden sus ocupaciones y cómo dan sentido a su existencia; a través de la intervención buscan fomentar la participación de sus clientes en ocupaciones significativas.

\section{Conclusiones}

Esta revisión ha evidenciado las diferencias presentes en las conceptualizaciones de ocupación propuestas en los Cadernos Brasileiros de Terapia Ocupacional hasta el año 2014. La presencia de artículos que discuten las cuestiones conceptuales vinculadas a la ocupación son muy menores en proporción a otros que describen prácticas o que abordan la TO Social, por ejemplo, lo que permitiría intuir cuáles son las prioridades en el desarrollo de la investigación brasileña, según cuestionamientos realizados por autoras como De Padua \& Feriotti (2013) y Feriotti (2017), quienes se han preguntado por la exclusión de reflexiones sobre ocupación en el contexto brasileño.

De esta manera, resulta interesante preguntarse si efectivamente la conceptualización de ocupación se configura como un tema relevante en Brasil y cómo aquello podría compararse con el resto de países de la región Latinoamericana.

\section{RefERENCIAS BibliográfiCAS}

Aguilera, R. (2014). ¿Revisión sistemática, revisión narrativa o metaanálisis? Revista de la Sociedad Española del Dolor, 27(6), 359-360. doi:10.4321/S1134-80462014000600010

Araújo, E., Guimarães, D. \& Di Napoli, M. (2013). Atividade, ação, fazer e ocupação: adiscussão dos termos na Terapia Ocupacional brasileira. Cadernos De Terapia Ocupacional da UFSCAR, 27(2), 243-254. Disponible en: http://dx.doi.org/10.4322/cto.2013.026

Caniglia, M. (2005). Terapia Ocupacional: Um enfoque disciplinar. Belo Horizonte,Brasil: Ophicina de Arte e Prosa.
Carleto, D. G., Cavalcanti, A.; Silva, M.; de Cruz, D. M. \& Andrade, V. S. (2010). Estrutura da prática da terapia ocupacional: domínio e processo. 2 ed. Revista Triângulo, Uberaba, 3(2), 57-147. Disponible en: http://seer.uftm.edu.br/revistaeletronica/index.php/ revistatriangulo/article/view/150/177

Carrasco, J. \& Olivares, D. (2008). Haciendo camino al andar: construcción y comprensión de la Ocupación para la investigación y práctica de la Terapia Ocupacional. Revista Chilena de Terapia Ocupacional, 8, 5-16. doi:10.5354/0717-5346.2008.55

Da Silva, M. \& Dornelas, A. (2014). Desempenho Funcional De Crianças Com Desnutrição Crônica Na Faixa Etária De Um A Três Anos. Cadernos de Terapia Ocupacional da UFSCAR, 22(2), 327-334. http://dx.doi.org/10.4322/cto.2014.054

De Padúa, E. M. M., \& Feriotti, M. L. (Eds.). (2013). Terapia Ocupacional e Complexidade. Práticas multidimensionais. Curitiba, Brasil: Editora CRV.

Echeverría, R. T. A., Ortega, L. T. P. \& Riquelme, E. V. (2009). Explorando necesidades ocupacionales: un estudio de caso. Revista Chilena de Terapia Ocupacional, Santiago, 9, 117-131.

Francisco, B. R. (2004). Terapia Ocupacional. São Paulo: Ed. Papirus.

Feriotti, M. L. (2017). Construcción de la identidad profesional del terapeuta ocupacional en el marco epistemológico actual: Una mirada particular desde Brasil. Revista Terapia Ocupacional Galicia, 14(25), 17-31. Disponible en: http://www.revistatog.com/num25/ pdfs/100a\%C3\%B1osdeterapia1.pdf

Gómez, S. (2003). La ocupación y su significado como factor influyente de la identidad personal. Revista Chilena de Terapia Ocupacional, 3, 43-47. doi:10.5354/0717-5346.2003.149

Gómez, S. (2006). Equilibrio y organización de la rutina diaria. Revista Chilena de Terapia Ocupacional, 6, 47-54. Doi:105354/07176767.2006.111

Imperatore, E., \& Henny, E. (2002). La filosofía de la terapia ocupacional, ciencia ocupacional e ideología: Una propuesta de interrelación. Revista Chilena de Terapia Ocupacional, 2. doi: 10.5354/0717-6767.2002.133

Kantartzis, S. \& Molineux, M. (2012). Understanding the discursive development of occupation: Historico-political perspectives. En: Whiteford, G.; Hocking, C. (Ed.). Occupational science: Society, inclusion, participation (pp. 38-53). Oxford: Blackwell Publishing Ltd.

Kiepek, N., \& Magalhaes, L. (2011). Addictions and Impulse Control Disorders as Occupation: A Selected Literature Review and Synthesis. Journal of Occupational Science, 18(3), 254-276. http:// dx.doi.org/10.1080/14427591.2011.581628 
Larson, E., Wood, W. \& Clark, F. (2005). Ciencia Ocupacional: desarrollo de la ocupación a través de una disciplina académica. En: Crepeau, E. B.; Cohn, E. S.; Schell, B. A. B. Willard \& Spackman: Terapia Ocupacional (pp. 16-26). Buenos Aires: Médica Panamericana.

Law, M., \& Baum, C. (1998). Evidence-based occupational therapy. Canadian Journal of Occupational Therapy, 65, 131-135. http:// dx.doi. org/10.1177/000841749806500301

Lima, C., Loureiro, A., Bentes, A., de Lima, A. \& Costa, B. (2013). O Valor Terapêutico Da Ação Humana E Suas Concepções Em Terapia Ocupacional. Cadernos de Terapia Ocupacional da UFSCAR, 27(1), 195-203. Disponible en http://dx.doi.org/10.4322/cto.2013.008

Magalhães, L. \& Galheigo, S. (2010). Enabling international communication among Brazilian occupational therapists: Seeking consensus on occupational terminology. Occupational Therapy International, 17(3), 113-124. http://dx.doi.org/10.1002/ oti.292

Magalhães, L. (2013). Ocupação e atividade: tendências e tensões conceituais na literatura anglófona da Terapia Ocupacional e da Ciência Ocupacional. Cadernos de Terapia Ocupacional da UFSCAR, 27(2): 255-263. Disponible en: http://dx.doi.org/10.4322/ cto. 2013.027

Martinelli, S. (2011). A importância de atividades de lazer na Terapia Ocupacional. Cadernos de Terapia Ocupacional da UFSCAR, 19(1): 111-118.

Monteiro, L., Ferreira, E., Cavaleiro, V. \& Costa, O. (2014). Sobre o significado das ocupações após o acidente por queimaduras. Cadernos de Terapia Ocupacional da UFSCAR, 22(2): 305-315. Disponible en: http://dx.doi.org/10.4322/cto.2014.052

Morrison, R., Gómez, S., Henny, E., Tapia, M. J., \& Rueda, L. (2017). Principal Approaches to Understanding Occupation and Occupational Science Found in the Chilean Journal of Occupational Therapy (2001-2012). Occupational therapy international. Disponible en: https://doi.org/10.1155/2017/5413628

Nelson, D. (1997). Why the profession of occupational therapy will flourish in the 21st Century. [The Eleanor Clarke Slagle Lecture] American Journal of Occupational Therapy, 57(1): 11-24

Nelson, D. L. \& Jonsson, H. (1999). Occupational terms across languages and countries. Journal of Occupational Science, 6(1), 42-47

Pierce, D. (2001). Untangling occupation and activity. American Journal of Occupational Therapy, 55(2), 138-146. http://dx.doi.org/10.10 80/14427591.2010.9686697

Prodinger, B. \& Stamm, T. (2012). The emergence of occupational science in Austria: An insider perspective. Journal ofOccupational Science, 19(2), 127-137. http://dx.doi.org/10.1080/14427591.2011.582833
Ramugondo E. L. \& Kronenberg, F. (2015). Explaining Collective Occupations from a Human Relations Perspective: Bridging the Individual-Collective Dichotomy. Journal of Occupational Science, 22(1): 3-16. doi:10.1080/14427591.2013.781920

Royeen, C. (2002). Occupation reconsidered. Occupational Therapy International, 9(2), 11-20.

Rueda, L. (2003). La Ocupación: ciencia y técnica de la terapia ocupacional. Revista Chilena de Terapia Ocupacional, 3, 1-5. doi:10.5354/0717-5346.2003.144

Stoffel, D. \& Nickel, R. (2013). A utilização da atividade como ferramenta no processo de intervenção do Terapeuta Ocupacional em reabilitação neurológica. Cadernos de Terapia Ocupacional da UFSCAR, 27(3): 617-622. Disponible em: http://dx.doi. org/10.4322/cto.2013.064

Torrico, P., Urrutia, D. \& Schliebener, M. (2008). ¿Qué significado le asignan un grupo de mujeres a las actividades para sí mismas? Revista Chilena de Terapia Ocupacional, 8, 17-25. doi:10.5354/07175346.2008 .57

Wilcock, A. A. (2007). Occupation and health: Are they one and the same? Journal of Occupational Science, 14(1), 3-8.http://dx.doi. org/10.1080/144 27591.2007.9686577 